ARTÍCULO DE REVISIÓN

\title{
Linfomas en la era COVID-19
}

\author{
Juan F. Zazueta-Pozos ${ }^{1 *}$, Silvia Rivas-Vera², Efreén Montaño-Figueroa ${ }^{1}$, Luis Villela-Martínez³ y \\ José Álvarez-Vera ${ }^{4}$ \\ 'Departamento de Hematología, Hospital General de México Dr. Eduardo Liceaga, Ciudad de México, Méx.; '2Departamento de Hematología, \\ Instituto Nacional de Cancerología, Ciudad de México, Méx.; ${ }^{3}$ Departamento de Hematología, Centro Médico Dr. Ignacio Chávez ISSSTESON, \\ Hermosillo, Son., ${ }^{4}$ Departamento de Hematología, Centro Médico Nacional 20 de Noviembre ISSSTE, Ciudad de México, Méx. México
}

\section{Resumen}

El coronavirus 2 del síndrome respiratorio agudo grave (SARS-CoV-2) y su traducción clínica, la enfermedad por coronavirus 2019 (COVID-19), representan una enfermedad con manifestaciones respiratorias potencialmente fatales. Actualmente existen aproximadamente 12,700,000 personas afectadas por esta virus, el cual ha ocasionado 561,517 muertes en el mundo. Los pacientes con diagnóstico de linfoma, al igual que otros pacientes con cáncer activo, presentan compromiso inmunitario, ya sea por su propia patología o debido al tratamiento que reciben, por lo que son especialmente susceptibles a desarrollar cuadros graves de COVID-19. La transmisión comunitaria del SARS-CoV-2 dificulta el acceso al sistema de salud y, por ende, el seguimiento estricto que requieren lo pacientes bajo tratamiento oncológico. En la etapa en la que nos encontramos actualmente, la transmisión global de la infección por SARS-CoV-2 continúa en ascenso, por lo que un control epidemiológico cercano es poco probable. Ante este contexto, surge la necesidad de establecer guías de tratamiento de pacientes con neoplasias hematológicas.

PALABRAS CLAVE: Linfoma no Hodgkin. Linfoma difuso de células grandes B. Linfoma del manto. Linfoma folicular. SARSCoV-2. Coronavirus.

\section{Lymphoma and COVID-19}

\section{Abstract}

The SARS-CoV-2 virus and its clinical translation COVID-19 represent a disease with potentially fatal respiratory manifestations. Currently, there are approximately 12,700,000 people affected by this virus, which has caused 561,517 deaths worldwide. Patients with a diagnosis of lymphoma, like other patients with active cancer, have immune compromise either due to their own pathology or due to the treatment they receive, making them especially susceptible to developing severe cases of COVID-19. The community transmission of SARS-CoV-2 hinders access to the health system and, therefore, the strict monitoring required by patients undergoing cancer treatment. At the stage we are currently in, global transmission of SARS-CoV-2 infection continues to rise, making close epidemiological control unlikely. In this context, the need arises to establish guidelines for the treatment of patients with hematological malignancies.

KEY WORDS: Non-Hodgkin lymphoma. Diffuse large B cell lymphoma. Mantle cell lymphoma. Follicular lymphoma. SARSCoV-2. Coronavirus. 


\section{Introducción}

Los pacientes con diagnóstico de linfoma, al igual que otros pacientes con cáncer activo, presentan compromiso inmunitario, ya sea por su propia patología o debido al tratamiento que reciben, por lo que son especialmente susceptibles a desarrollar cuadros graves de COVID-19. La transmisión comunitaria del SARS-CoV-2 dificulta el acceso al sistema de salud $y$, por ende, el seguimiento estricto que requieren lo pacientes bajo tratamiento oncológico., ${ }^{1,2}$ Los linfomas no Hodgkin (LNH) son un grupo heterogéneo de patologías neoplásicas con un espectro de comportamiento amplio, el cual incluye padecimientos indolentes hasta neoplasias altamente agresivas. Las características clínicas y su comportamiento están estrechamente ligados a la etapa de maduración del linfocito, del cual se originan ${ }^{3}$.

El Proyecto internacional para la clasificación del Linfoma no Hodgkin reveló que los LNH de estirpe B son más comunes, y demostró que, en países de mayor desarrollo económico, la incidencia de linfomas indolentes es mayor, mientras que en países en vías de desarrollo, existe una mayor incidencia de linfomas agresivos y de estirpe $T$ y natural killer.

El tratamiento inicial de los LNH depende de diversos factores. La decisión clínica para inicio de tratamiento depende del comportamiento inicial de la enfermedad y de su evolución natural en ausencia de tratamiento. En este sentido, los linfomas indolentes usualmente afectan al paciente en el transcurso de varios años, los linfomas agresivos en el transcurso de meses y aquellos con características leucémicas en el transcurso de semanas ${ }^{5}$.

Cerca del $90 \%$ de los pacientes con linfomas indolentes se presentan con estadios clínicos avanzados. Con los tratamientos actuales, este tipo de linfomas se consideran incurables, por lo que el tratamiento consiste en cursos cortos de quimio-inmunoterapia, para lograr remisiones temporales libres de tratamiento ${ }^{4}$. En contraste, los linfomas agresivos son tratados con la premisa de curación, con excepción del linfoma de células del manto. Por lo tanto, para los LNH difusos de células grandes, los linfomas T periféricos y otros linfomas agresivos, los esquemas de tratamiento incluyen quimioterapia combinada \pm radioterapia local 0 a sitio involucrado, así como el empleo de anticuerpos monoclonales en subtipos específicos. Aproximadamente un $75 \%$ de pacientes con estadios localizados y subtipos agresivos pueden lograr la cura con las terapias disponibles actualmente ${ }^{6}$.

En el caso de los pacientes con $\mathrm{LNH}$, la necesidad de iniciar, continuar o diferir un tratamiento en el contexto de la pandemia por SARS-CoV-2 ha originado una serie de recomendaciones por la comunidad internacional, las cuales tienen la finalidad de establecer un algoritmo replicable en el tratamiento de estos pacientes. Los puntos más importantes para considerar incluyen:

- El empleo de quimioterapia combinada en relación con la curva epidemiológica de cada región.

- El uso de inmunoterapia, incluidos los anticuerpos monoclonales para linfomas agresivos e indolentes.

- Uso de esquemas mieloablativos en pacientes linfomas agresivos.

- El empleo de mantenimiento con anticuerpos monoclonales en pacientes con linfomas indolentes.

- El papel de la radioterapia de consolidación.

- Esquemas de tratamiento en el contexto de refractariedad/recaída en linfomas agresivos.

- El papel del trasplante de células progenitoras hematopoyéticas autólogo en la situación clínica indicada para cada patología.

- El uso de profilaxis con factor estimulante de colonias de granulocitos y terapia antimicrobiana como profilaxis primaria o secundaria.

Con la finalidad de establecer el abordaje terapéutico más adecuado, se emiten las siguientes recomendaciones en sincronía con las establecidas a nivel mundial.

\section{Recomendaciones para pacientes con neoplasias linfoides durante la pandemia de COVID-19}

El estado de la enfermedad en el caso de estas neoplasias es un factor importante para la decisión terapéutica. Una recomendación muy generalizada para los pacientes con neoplasias linfoides en remisión es posponer las consultas y de ser posible realizar monitoreo por vía telefónica y virtual.

En general las recomendaciones internacionales dependen de la agresividad de los $\mathrm{LNH}$, considerando agresivos a los linfomas: difusos de células grandes, primario mediastinal, plasmablástico, Burkitt, linfoplasmablástico, de células del manto y linfomas periféricos de células $T$. En las neoplasias indolentes se incluyen el linfoma folicular, los linfomas de la zona 
marginal, de células pequeñas, etc. El linfoma de Hodgkin (LH) se considera de forma independiente.

Tomando en consideración la frecuencia de los linfomas, se presentarán recomendaciones generales para los principales linfomas agresivos que afectan a nuestra población, de acuerdo con la clasificación comentada previamente

\section{Recomendaciones para pacientes con linfomas agresivos durante la pandemia de COVID 19}

\section{Linfoma no Hodgkin difuso de células grandes B}

Los linfomas no Hodgkin difusos de células grandes B (LNH DCGB) constituyen entre el 30 y el $58 \%$ de Ios $\mathrm{LNH}$ en algunas series publicadas. La incidencia en Europa es de 3.8/100,000 casos/año7. Las estrategias de tratamiento deben establecerse de acuerdo con varios factores, dentro de los cuales destacan la edad, el índice pronóstico internacional, la clasificación citogenética/molecular y la capacidad de inclusión a ensayos clínicos ${ }^{8}$.

La introducción de rituximab, anticuerpo monoclonal anti-CD20, como terapia adyuvante al esquema clásico de quimioterapia basado en antraciclinas, esquema CHOP (ciclofosfamida, prednisona, vincristina, doxorubicina), ha sido considerado uno de los avances más relevantes en el tratamiento del LNH DCGB de los últimos 20 años. Dicha estrategia demostró mejoría en los resultados del tratamiento de pacientes con esta neoplasia, tanto en ensayos clínicos prospectivos aleatorizados como en estudios observacionales ${ }^{9}$. Por lo tanto, el esquema R-CHOP es el tratamiento estándar para el LNH DCGB. Diversos estudios han planteado la necesidad de nuevos tratamientos para pacientes con variantes agresivas (doble expresores y doble o triple $h i t)^{10}$. Dichas estrategias se basan en la adición de anticuerpos monoclonales, terapias dirigidas e incremento de dosis para vencer el riesgo otorgado por las variantes identificadas como adversas.

\section{Linfoma de células del manto}

El linfoma de células del manto (LCM) representa entre el 3 y el $10 \%$ de los LNH. Es una neoplasia linfoproliferativa con un carácter generalmente agresivo, aunque una heterogeneidad en su comportamiento inicial es frecuente ${ }^{11}$.
El curso clínico del LCM varía de acuerdo con la presentación inicial, por lo que dentro del abordaje inicial es imperativo realizar una adecuada caracterización de la enfermedad en sus variantes pleomórfica, blastoide y de linfocitos pequeños con una presentación generalmente asintomática.

La gran mayoría de pacientes $(70-80 \%)$ se presentan con linfadenopatía progresiva o síntomas relacionados a involucro extranodal, ambas con requerimiento de tratamiento sistémico inicial.

Por otro lado solo un $20-30 \%$ podrán estar bajo vigilancia y seguimiento, estrategia conocida como «ver y esperar»" ${ }^{12}$.

Diversas recomendaciones en cuanto al tratamiento de pacientes con diagnóstico reciente de LNH agresivos (incluidos LNH DCGB y LCM) han surgido con el objetivo de orientar el manejo y seguimiento de estos pacientes durante la evolución de la pandemia por COVID-19.

- Pacientes de reciente diagnóstico:

- Reducir el número de visitas médicas de pacientes ambulatorios. De ser posible, utilizar tecnología de videoconferencia para seguimiento y evaluación de pacientes.

- Las recomendaciones actuales del grupo Americano, representado por la Sociedad Americana de Hematología (ASH), sugieren mantener la expectativa de curación para los pacientes con LNH DCGB y otros linfomas agresivos mediante el inicio expedito de tratamiento con R-CHOP o el tratamiento de primera línea correspondiente ${ }^{13}$.

- Para linfomas de alto grado, como los linfomas doble o triple hit, el uso de esquemas infusionales como R-DA-EPOCH (rituximab, fosfato de etopósido, prednisona, sulfato de vincristina, ciclofosfamida, clorhidrato de doxorubicina) debe ser evaluado de manera individualizada ante el riesgo de mielosupresión atribuible a dichos esquemas, así como la necesidad de hospitalización para su administración.

- No retrasar el tratamiento de linfomas agresivos, como linfoma de Burkitt, linfoma linfoblástico y linfoma plasmablástico.

- El empleo de anticuerpos monoclonales (rituximab) debe individualizarse de acuerdo con los factores de riesgo intrínsecos (comorbilidades) y extrínsecos (curva epidemiológica local) en pacientes con LNH agresivos ya que, de no existir contraindicaciones, se sugiere otorgar el beneficio de la inmunoterapia 
adyuvante al tratamiento con quimioterapia convencional.

- Aquellos centros que tengan la capacidad técnica de utilizar tratamientos infusionales ambulatorios deben optar por esta modalidad.

- Por su parte, el Grupo Español de Linfomas y Trasplante de Médula Ósea (GELTAMO) insta a mantener el tratamiento de pacientes con esta patología, tras una adecuada valoración del riesgo/beneficio tras un abordaje individualizado. De la misma manera, el grupo español mantiene la postura de consolidar el tratamiento con trasplante autólogo de células progenitores hematopoyéticas en el contexto de recaída de linfomas agresivos.

- La Sociedad Europea de Oncología Médica (ESMO) sugiere continuar con los modelos de tratamiento actuales, proponiendo un cambio en el esquema de visita médica, hacia modelos externos (telemedicina).

- Para estadios clínicos localizados, se sugiere el empleo de cuatro ciclos de R-CHOP en lugar de tratamiento multimodal (quimioterapia + radioterapia), con la finalidad de reducir el número y frecuencia de visitas hospitalarias.

- No realizar o retrasar los trasplantes alogénicos previamente planeados.

- Emplear factores estimulantes de colonias de granulocitos a fin de evitar periodos de neutropenia durante el tratamiento.

- Considerar el seguimiento mediante la premisa «ver y esperar» en pacientes con LCM variante no nodal-no blastoide asintomáticos.

- En pacientes con LCM que se consideren aptos para tratamiento intensivo y consolidación con trasplante autólogo de células progenitoras hematopoyéticas, se sugiere iniciar con esquemas basados en citarabina, debido a los excelentes resultados en supervivencia libre de progresión y supervivencia global. La movilización de progenitores hematopoyéticos y su cosecha se debe realizar tras tres o cuatro ciclos de tratamiento.

- Se sugiere diferir el mantenimiento con rituximab en pacientes con LCM.

- Valorar el uso de formulaciones subcutáneas (rituximab) para evitar tiempos prolongados en clínicas de infusión.

- Se sugiere utilizar el central nervous system international prognostic index (CNS-IPI) para establecer que pacientes tienen alto riesgo de involucro del sistema nervioso central, quienes deberán recibir profilaxis de manera obligatoria con metotrexato intravenoso o de acuerdo con las guías locales de tratamiento.

- Posponer todas las visitas médicas de los pacientes en remisión y en quienes no se tiene contemplado alguna intervención terapéutica.

- Pacientes en recaída/refractarios:

- Los tratamientos de rescate en el contexto de refractariedad/recaída deben individualizarse, dando mayor énfasis en continuar esquemas de quimioterapia intensiva solo con fines curativos. En pacientes con intención paliativa, se recomienda aplazar las visitas médicas y optar por dar tratamientos ambulatorios.

- Retrasar el empleo de radioterapia con fines paliativos.

- Para pacientes con linfoma del manto en recaída, se deben optar por el uso de terapias orales, incluyendo inhibidores de la tirosina cinasa de Bruton y lenalidomida.

- Los pacientes bajo tratamiento con quimioterapia deben recibir factor estimulante de colonias de granulocitos para evitar periodos prolongados de neutropenia.

\section{Linfoma de células $T$ periféricas}

- Los linfomas de células T periféricas en nuestro país representan aproximadamente un $15-18 \%$ de todos los linfomas. Por su agresividad requieren inicio inmediato del tratamiento. Hasta el momento no se ha encontrado un esquema óptimo, aunque en la mayoría de los centros se utilizan esquemas basados en $\mathrm{CHOP} \pm$ etopósido para los LCTP ganglionares ${ }^{14}$. En el caso de los linfomas de células T/NK los esquemas recientes se basan en el uso de L-asparaginasa ${ }^{15}$. El tratamiento para el Linfoma de anaplásico de células grandes ALK-negativo tampoco está definido. Existe acuerdo en relación con la terapia de consolidación con trasplante hematopoyético en este grupo de pacientes ${ }^{16}$.

- Aspectos prácticos:

- Evitar el retraso en el inicio del tratamiento.

- Se recomienda el empleo $\mathrm{CHOP} \pm$ etopósido para la mayoría de los LCTP ganglionares.

- Considerar retrasar el trasplante hematopoyético en pacientes en remisión completa después de explicarle y riesgos y beneficios de este.

- Posponer citas médicas de pacientes en remisión o de pacientes en los que no haya cambio 
inmediato. Se sugieren consultas telefónicas o virtuales.

\section{Recomendaciones para pacientes con linfomas indolentes durante pandemia COVID 19}

\section{Linfoma folicular}

El linfoma folicular (LF) es una neoplasia linfoproliferativa originada de tejido linfoide que muestra diferenciación del centro germinal. Representa aproximadamente el $5 \%$ de las neoplasias hematológicas y un $20-25 \%$ de todos los $\mathrm{LNH}$ en población caucásica ${ }^{14}$

El curso del linfoma folicular suele ser asintomático, por lo que la mayoría de los pacientes se presentan con estadios avanzados de la enfermedad, con solo un $20-30 \%$ presentándose en estadios I o II. El tratamiento de las etapas tempranas se basa en el uso de radioterapia con o sin terapia sistémica, lo cual otorga remisiones completas duraderas en aproximadamente un $50 \%$ de los pacientes ${ }^{15}$. En algunos casos, la conducta de «ver y esperar» puede ser válida, especialmente en pacientes con estadios localizados con múltiples comorbilidades ${ }^{16}$.

Para pacientes con estadios avanzados con baja carga tumoral, la decisión de iniciar tratamiento deberá individualizarse, incluso en este contexto la terapia «ver y esperar» es válida. El empleo de monoterapia con rituximab es una estrategia que ha mostrado resultados favorables en este escenario, logrando diferir el inicio de tratamiento subsecuente por un promedio de ocho años ${ }^{17}$. Por otro lado, los pacientes con estados avanzados y alta carga tumoral son usualmente tratados con esquemas de quimio-inmunoterapia, siendo los más empleados R-CHOP y R-bendamustina. El mantenimiento con rituximab ha demostrado mejorar la supervivencia libre de enfermedad, aunque no repercute en la supervivencia global, por lo tanto su uso debe ser evaluado de manera individualizada de acuerdo con los riesgos y comorbilidades de cada paciente.

- Pacientes de reciente diagnóstico:

- Valorar el empleo de conducta «ver y esperar» para pacientes con estadios localizados o avanzados con baja carga tumoral.

- Se sugiere utilizar los criterios GELF (Groupe d'Étude des Lymphomas Folliculaires) para valorar el inicio de tratamiento sistémico ${ }^{18}$.
- Utilizar monoterapia con rituximab en pacientes candidatos a tratamiento, de acuerdo con la curva epidemiológica local de casos activos a infección por SARS-CoV-2.

- Se sugiere mantener el uso de radioterapia con fines curativos para pacientes con enfermedad localizada 0 estadios clínicos tempranos.

- Emplear factores estimulantes de colonias de granulocitos a fin de evitar periodos de neutropenia durante el tratamiento.

- El mantenimiento con terapia anti-CD20 debe evitarse durante la pandemia de COVID-19, para evitar inmunosupresión prolongada, estancias prolongadas en centros de infusión y visitas frecuentes al hospital.

- Se sugiere diferir las terapias de consolidación o rescate con dosis altas de quimioterapia e infusión de progenitores hematopoyéticas.

- Valorar el uso de formulaciones subcutáneas (rituximab) para evitar tiempos prolongados en clínicas de infusión.

- Posponer todas las visitas médicas de los pacientes en remisión y en quienes no se tiene contemplado alguna intervención terapéutica.

- Pacientes en recaída/refractarios:

- Los tratamientos de rescate en el contexto de refractariedad/recaída deben individualizarse, dando mayor énfasis en continuar esquemas de quimioterapia intensiva solo con fines curativos. En pacientes con intención paliativa, se recomienda aplazar las visitas médicas y optar por dar tratamientos ambulatorios.

- En caso de seropositividad para COVID-19, el inicio del tratamiento se debe diferir hasta contar con el protocolo de seronegatividad correspondiente a cada institución. Si un paciente bajo tratamiento cuenta con una prueba positiva para infección por SARS-CoV-2, se deberá valorar la suspensión del tratamiento y vigilancia estrecha del caso.

- En el contexto de linfomas indolentes, con baja probabilidad de cura, el manejo de pacientes en recaída debe enfocarse al control de la enfermedad en casos agresivos y a vigilancia en casos con baja carga tumoral.

- Retrasar el empleo de radioterapia con fines paliativos.

- Los pacientes bajo tratamiento con quimioterapia deben recibir factor estimulante de 
colonias de granulocitos para evitar periodos prolongados de neutropenia.

\section{Linfoma de Hodgkin}

El LH es altamente curable con las estrategias de tratamiento actuales ${ }^{19}$. El ABVD (doxorubicina, bleomicina, vinblastina y dacarbazina), una terapia tolerable y efectiva, es el régimen más utilizado en todo el mundo ${ }^{20}$. Hay una baja probabilidad de neutropenia febril con ABVD, y los pacientes generalmente tienen una baja utilización de recursos médicos ${ }^{21}$. Aunque existen esquemas más intensivos como BEACOPP escalado (bleomicina, etopósido, doxorubicina, ciclofosfamida, vincristina, procarbazina y prednisona) ${ }^{22}$ y más recientemente la adición de nuevas terapias como brentuximab vedotin ${ }^{23}$, es difícil equilibrar los riesgos y beneficios de un régimen más intensivo en momentos de agotamiento de los recursos médicos. Sin embargo, dado que no existe un consenso claro sobre el papel de los regímenes más intensivos que consumen más recursos, el ABVD parece una buena opción como tratamiento inicial. La radioterapia para la enfermedad en etapa temprana generalmente se aplica, pero se puede omitir si no está disponible con un impacto del 5 al $8 \%$ en supervivencia libre de progresión ${ }^{24}$. Una terapia guiada por PET/CT (tomografía por emisión de positrones/tomografía computarizada) puede ayudar a identificar a los pacientes en quienes se puede omitir la radioterapia ${ }^{25}$.

En el caso de recaída se recomienda preferir el rescate ambulatorio, incluidos los regímenes basados en gemcitabina. El trasplante no debe retrasarse a menos que haya una falta crítica de camas disponibles, debido a la posibilidad de cura. Si se indican inhibidores del punto de control (ICP), se debe preferir la dosificación de cuatro y seis semanas para nivolumab $^{26}$ y pembrolizumab ${ }^{27}$ cuando sea posible.

Puntos prácticos:

- Evitar el retraso del tratamiento del LH a menos que sea extremadamente necesario.

- El ABVD es actualmente el régimen más utilizado. No existe consenso sobre la elección de ABVD y BV-AVD para etapas avanzadas de la enfermedad.

- Evitar los regímenes más intensivos (BEACOPP).

- Podría omitirse la radioterapia si no está disponible, a un costo del 6 al $8 \%$ de control de la enfermedad. Los pacientes pueden beneficiarse de seis ciclos de $A B V D$ si no se usa radioterapia.
Considerar usar una estrategia guiada por PET y discuta los riesgos y beneficios con los pacientes.

- Preferir el rescate ambulatorio.

- Retrasar trasplante de precursores hematopoyéticos solo en casos extremos.

- No hay una recomendación actual para los cambios de consolidación con BV durante la pandemia de COVID-19. La interrupción o demora del mantenimiento de BV debe discutirse individualmente.

- Deben intentarse las dosis de las semanas de los ICP cuatro y seis siempre que sea posible.

- Posponer citas médicas de pacientes en remisión completa o para pacientes en los que no se espera un cambio inmediato en la terapia. Se recomienda la consulta/asesoramiento virtual.

\section{Financiamiento}

Los autores no recibieron ningún financiamiento específico para este trabajo.

\section{Conflicto de intereses}

Los autores declaran que no existe conflicto de intereses.

\section{Responsabilidades éticas}

Protección de personas y animales. Los autores declaran que para esta investigación no se han realizado experimentos en seres humanos ni en animales.

Confidencialidad de los datos. Los autores declaran que en este artículo no aparecen datos de pacientes.

Derecho a la privacidad y consentimiento informado. Los autores declaran que en este artículo no aparecen datos de pacientes.

\section{Bibliografía}

1. World Health Organization. Coronavirus disease (COVID-19) [Internet]. World Health Organization [consultado: 12 julio 2020]. Disponible en: https://www.who.int/emergencies/diseases/novel-coronavirus-2019

2. Weinkove R, Mcquilten ZK, Adler J, Agar MR, Blyth E, Cheng AC, et al. Managing haematology and oncology patients during the COVID-19 pandemic: interim consensus guidance. Med J Aust. 2020;212(10):24.

3. Armitage JO, Gascoyne RD, Lunning MA, Cavalli F. Non-Hodgkin lymphoma. Lancet. 2017;390(10091):298-310.

4. Connors JM. Non-Hodgkin lymphoma: the clinician's perspective - a view from the receiving end. Mod Pathol. 2012;26:S111-S118.

5. Perry AM, Diebold J, Nathwani BN, Maclennan KA, Müller-Hermelink HK, Bast M, et al. Non-Hodgkin lymphoma in the developing world: Review of 4539 cases from the international Non-Hodgkin Lymphoma Classification Project. Haematologica. 2016;101(10):1244-50. 
6. Coiffier B, Thieblemont C, van den Neste E, Lepeu G, Plantier I, Castaigne $\mathrm{S}$, et al. Long-term outcome of patients in the LNH-98.5 trial, the first randomized study comparing rituximab-CHOP to standard $\mathrm{CHOP}$ chemotherapy in DLBCL patients: A study by the Groupe d'Etudes des Lymphomes de l'Adulte. Blood. 2010;116(12):2040-5.

7. Sant M, Allemani C, Tereanu C, De Angelis R, Capocaccia R, Visser O et al. Incidence of hematologic malignancies in Europe by morphologic subtype: results of the HAEMACARE project. Blood. 2010;116(19):3724-34

8. Tilly H, Gomes Da Silva M, Vitolo U, Jack A, Meignan M, Lopez-Guillermo A, et al. Diffuse large B-cell lymphoma (DLBCL): ESMO Clinica Practice Guidelines for diagnosis, treatment and follow-up. Ann Oncol. 2015;26(Suppl 5):v116-25.

9. Coiffier B, Lepage E, Briere J, Herbrecht R, Tilly H, Bouabdallah R, et al. CHOP chemotherapy plus rituximab compared with $\mathrm{CHOP}$ alone in elderly patients with diffuse large-B-cell lymphoma. N Engl J Med. 2002;346(4):235-42

10. Landsburg DJ, Petrich AM, Abramson JS, Sohani AR, Press O, Cassaday $\mathrm{R}$, et al. Impact of oncogene rearrangement patterns on outcomes in double-hit non-Hodgkin lymphoma. Cancer. 2016;122(4):559-64.

11. Teras LR, De Santis CE, Cerhan JR, Morton LM, Jemal A, Flowers CR. 2016 US Iymphoid malignancy statistics by World Health Organization subtypes. CA Cancer J Clin. 2016:66(6):443-59.

12. Abrisqueta P, Scott DW, Slack GW, Steidl C, Mottok A, Gascoyne RD et al. Observation as the initial management strategy in patients with mantle cell lymphoma. Ann Oncol. 2017;28:2489-95.

13. COVID-19 and aggressive lymphoma [Internet]. Hematology. org [citado: 12 julio 2020]. Disponible en: https://www.hematology.org/covid-19/covid-19-and-aggressive-lymphoma

14. Gleeson M, Peckitt $C$, To YM, Edwards L, Oates J, Wotherspoon A, et al. CHOP versus GEM-P in previously untreated patients with periphera T-cell lymphoma (CHEMO-T): a phase 2, multicentre, randomised, open-label trial. Lancet Haematol. 2018;5(5):e190-200. doi: 10.1016/ S2352-3026(18)30039-5.

15. Yamaguchi $M$, Tobinai $K$, Oguchi M, Ishizuka N, Kobayashi $Y$, Isobe $Y$, et al. Concurrent chemoradiotherapy for localized nasal natural kiIler/T-cell lymphoma: an updated analysis of the Japan clinical oncology group study JCOG0211. J Clin Oncol. 2012:30(32);4044-6.

16. D'Amore F, Relander T, Lauritzsen GF, Jantunen E, Hagberg H, Anderson $\mathrm{H}$, et al. Up-front autologous stem-cell transplantation in peripheral T-cell lymphoma: NLG-T-01. J Clin Oncol. 2012;30(25):3093-9.
17. Ardeshna KM, Qian W, Smith P, Braganca N, Lowry L, Patrick P, et al. Rituximab versus a watch-and-wait approach in patients with advanced-stage, asymptomatic, non-bulky follicular lymphoma: an open-label randomised phase 3 trial. Lancet Oncol. 2014;15(4):424-35.

18. Brice P, Bastion Y, Lepage E, Brousse N, Haïoun C, Moreau P, et al. Comparison in low-tumor-burden follicular lymphomas between an initial no-treatment policy, prednimustine, or interferon alfa: A randomized study from the Groupe d'Etude des Lymphomes Folliculaires. J Clin Oncol. 1997;15(3):1110-7.

19. Townsend W, Linch D. Hodgkin's lymphoma in adults. Lancet. 2012;380:836-47.

20. Longley J, Johnson PWM. Options for first line therapy of Hodgkin lymphoma. Hematol Oncol. 2019;37(S1):82-6.

21. Boleti E, Mead GM. ABVD for Hodgkin's lymphoma: full-dose chemotherapy without dose reductions or growth factors. Ann Oncol. 2007; 18(2):376-80

22. Skoetz N, Will A, Monsef I, Brillant C, Engert A, von Tresckow B. Comparison of first-line chemotherapy including escalated BEACOPP versus chemotherapy including ABVD for people with early unfavourable or advanced stage Hodgkin lymphoma. Cochrane Database Syst Rev. 2017;5(5):CD007941.

23. Connors JM, Jurczak W, Straus DJ, Ansell SM, Kim WS, Gallamini A, et al. Brentuximab vedotin with chemotherapy for stage III or IV Hodgkin's lymphoma. N Engl J Med. 2018;378(4):331-44.

24. Meyer RM, Gospodarowicz MK, Connors JM, Pearcey RG, Wells WA, Winter JN, et al. ABVD alone versus radiation-based therapy in limited-stage Hodgkin's lymphoma. N Engl J Med. 2012;366(5):399-408.

25. Radford J, Illidge T, Counsell N, Hancock B, Pettengell R, Johnson P, et al. Results of a Trial of PET-directed therapy for early-stage Hodgkin's lymphoma. N Engl J Med. 2015;372(17):1598-607.

26. Bi Y, Liu J, Furmanski B, Zhao H, Yu J, Osgood C, et al. Model-informed drug development approach supporting approval of the 4-week (Q4W) dosing schedule for nivolumab (Opdivo) across multiple indications: a regulatory perspective. Ann Oncol. 2019;30(4):644-51.

27. Lala M, Li M, Sinha V, de Alwis D, Chartash E, Jain L. A six-weekly (Q6W) dosing schedule for pembrolizumab based on an exposure-response (E-R) evaluation using modeling and simulation. J Clin Oncol. 2018;36(15_suppl):3062. 